\title{
ENERGY EFFICIENCY OF PARALLEL MULTICORE PROGRAMS
}

\author{
DAVOR DAVIDOVIĆ* MATJAŽ DEPOLLI $\stackrel{*}{*}$ TOMISLAV LIPIĆ* KAROLJ SKALA* AND ROMAN TROBEC ${ }^{\dagger}$
}

\begin{abstract}
The increasing energy consumption of large-scale high performance resources raises technical and economical concerns. A reduction of consumed energy in multicore systems is possible to some extent with an optimized usage of computing and memory resources that is tailored to specific HPC applications. The essential step towards more sustainable consumption of energy is its reliable measurements for each component of the system and selection of optimally configured resources for specific applications. This paper briefly surveys the current approaches for measuring and profiling power consumption in large scale systems. Then, a practical case study of a real-time power measurement of multicore computing system is presented on two real HPC applications: maximum clique algorithm and numerical weather prediction model. We assume that the computing resources are allocated in a HPC cloud on a pay-per-use basis. The measurements demonstrate that the minimal energy is consumed when all available cores (up to the scaling limit for a particular application) are used on their maximal frequencies and with threads binded to the cores.
\end{abstract}

Key words: Energy efficiency, high-performance, maximum clique, numerical weather prediction

AMS subject classifications. 65Y05, 68U20

1. Introduction. Nowadays, a large number of hard problems is being solved efficiently on large-scale computing systems such as clusters, grids and clouds. The main building blocks of such systems are highperformance computing (HPC) clusters, since they are furthermore embedded both into grid computing systems as well as cloud computing systems [1]. A great advantage of Graphical Processor Unit (GPU) accelerators over multicore platforms was demonstrated in terms of energy efficiency [2] with an overall energy consumption for an order of magnitude smaller for the GPUs. Inside the clusters, the many-core architectures, such as GPUs [3], as well as even more specialized data-flow computers [4] are getting an increasingly large supporting role but the main processing is still performed by the multi-core main processors because of simple programming model. In this research, we analyze the energy consumption profile of the processors on a two processor multi-core computer, while varying its workload.

With the increasing number of components in large-scale computing systems the energy consumption is steeply increasing [5]. In computer systems, energy (measured in Joules) is represented as the electricity resource that can power the hardware components to do computation. On the other hand, power is the amount of energy consumed per unit time (measured in Joules per second or Watts). The ever-increasing energy consumption of large-scale computing systems causes higher operation costs (e.g. electricity bills) and has negative environmental impacts (e.g. carbon dioxide emissions). Therefore, when designing large-scale computing systems, the focus is often shifting from performance improvements to energy efficiency.

Energy can be reduced at several levels of the distributed architecture: individually on each node or hardware component-level, at the middleware level, at the networking level, and at the application level [6, 7, 8, 9].

Suboptimal use of systems resources (e.g. over-provisioning) is the primary source of energy inefficiency at middleware layer $[6,7,10]$. In over-provisioned systems, components usually consume excessive power when they are relatively idle or underutilized. This is important from the user point of view, because pay-peruse approaches stimulate users to maximally exploit rented resources during the execution period. Energyproportional computing [11] introduces the concept of consuming energy proportional to resource utilization so that an idle or underutilized component consumes less energy. For improving the energy efficiency at middleware layer, energy-aware resource and workload management algorithms are utilized [10]. Increased network traffic in many bandwidth sensitive network applications results also in increased number of networking devices such as switches and routers and therefore in increased energy consumption. Thus, different approaches for new energy aware network protocols and network infrastructure, e.g., software defined networks and network functions virtualization, are developed also for improving energy efficiency at network layer [12, 13].

*Rudjer Bošković Institute, Centre for Informatics and Computing, Zagreb, Croatia

$\dagger$ Jožef Stefan Institute, Department of Communication Systems, Ljubljana, Slovenia 
A processor is a component that dominates the energy consumption of each node. Thus, most of techniques are designed to reduce energy consumption of the processor [14, 15, 16]. Most of the current processors are implemented with dynamic voltage and frequency scaling regulators to optimally adjust the voltage/frequency at run-time according to the workload behavior. Paper [17] focuses on the dynamic control of the voltage regulators in a chip multicore processors platform. Results from detailed simulations based on realistic experimental setups demonstrate up to $9 \%$ total energy saving. In [18] a minimum energy voltage scheduling on a single multicore processor with software controlled dynamic voltage scaling is proposed. Another hardware approach for improving power management for multi-core systems is with implementation of multiple voltage islands [19]. Different frequency scaling schemes were investigated in the past that minimize the total energy consumption. The problem is even more critical in the real-time applications scheduling with pre-specified tasks' deadlines [20].

The energy consumption heavily depends on the characteristic of applications. Some applications are computationally intensive, other are data intensive, while other are hybrid of both. Energy-aware programming models, regarding various types of workloads and architectures, are required to develop energy efficient applications [21, 22]. Programming approaches, based on optimal utilization of shared hardware on-chip resources, e.g. cache memory, memory bandwidth, threads or cores, for improved energy efficiency are becoming popular because of their simple implementation. In heterogeneous multicore processors that integrate CPU cores and data-parallel accelerators (GPU) last-level cache memory can be managed to minimize the energy consumption [23]. The dynamic assignment of thread-to-core can be particularly beneficial in saving the energy [24, 25].

These approaches are particularly important in the case of modern multi-core building blocks that have a lot of options for controlling the execution parameters, e.g., number of cores, system frequency, core binding, etc. The energy can be thus saved at all levels of large-scale architectures, even dynamically, depending on the application program characteristics.

In this research we experimentally test the effects of the workload, core binding strategies, and frequency scaling on energy requirements for two existing applications. We build on the previous research [26] with addition of a communication intensive use case that significantly influences energy consumption. Our approach does not require any change in the original source code of the applications and is thus instantly applicable on a wide range of applications without inspecting their code. Our findings suggest that modern multi-core architectures perform best both energy-wise and performance-wise when all of the cores are used, the frequency is not throttled and threads are not allowed to migrate between cores.

The paper starts with a brief survey of the current approaches for measuring and profiling power consumption in Sect. 2. In Sect. 3, two different use cases are presented together with the description of the experimental computer for software-based energy measurements. In Sect. 4, the obtained results are shown under various test conditions, which demonstrate significant differences in the consumed energy. The results are discussed in more details in Sect. 5 in order to deduce some useful rules for users of HPC cloud resources. The paper concludes with the summary of results and directions for further work.

2. Energy Measurement and Profiling Approaches. The essential step towards the energy-efficiency is accurate, reliable and continuous energy consumption measurement of the system components. Energy measurement and profiling approaches can be classified as hardware-based, software-based and hybrid. In the following subsections the basic characteristics of hardware-based and software-based approaches are outlined, based on two extensive survey papers [27, 28].

2.1. Hardware-based Energy Measurement. Hardware-based energy measurement approaches are implemented with various instruments or sensors that measure the electrical current or the consumed energy as functions of time on different computation, storage or networking segments such as CPUs, motherboards, servers or racks. An energy measurement obtained with meters is a direct and straight-forward approach. A digital multi-meter collects the voltage or the energy samples of the measured segment in pre-specified time intervals and sends the measurements through a data link connected to the data collection system. Such meters are mainly used to measure the direct current (DC) power and are connected between the power supply and the measured segment. On the other hand, there are other meters (e.g. "Watts UP" [29]) that can directly measure the power consumption on the alternating current (AC) lines. Another example of a simple meter with limited precision is a clamp meter that has a larger measurement range and can be used to measure the energy of segments with high currents. AC meters, however, only measure the power consumption on the system level. 
In their simplicity, multi-meters cannot be used when a higher control of the measurement process is required. In those cases, more complex devices have to be used that are often custom designed. In 2000, Viredaz et al. designed a platform called Itsy to measure the energy consumption of mobile devices [30]. Another example of a complex device to measure the energy consumption is a single board on computer called PLEB [31]. PLEB uses a design with a set of on-board current sensors, and an analogue-to-digital converter with a micro-controller to read the sensors. Unlike Itsy, which measures only the energy consumption of mobile devices, PLEB can be used to measure the energy of processors, memories, flash drivers, and I/O components. Precise and finegrained measuring is also available through PowerPack, a system for power profiling on typical clusters or other distributed systems [32]. PowerPack measures power on system DC power lines and then maps it to individual components of the system, using computer models. It also measures AC power consumption to derive the efficiency of AC to DC conversion. All measurement are processed on a separate computer to ensure the power profiling does not impact the system under test. PowerPack profiling is enabled by the provided API calls, that link the application under test to control and communicate with the meter control process and thus correlate the power profiles with the application source code. Since PowerPack uses both hardware- and software-based methods of measurements it is actually a hybrid meter.

2.2. Software-based Energy Measurement. The disadvantages of hardware-based energy measurement techniques are in their dependence on the expensive sensing components and complex additional hardware design. Considering the expensive and cumbersome setup, they are also not well suited for online monitoring and designing of energy-aware policies. In contrary, software-based approaches can be used to supply more fine-grained online information, although with reduced accuracy. They are more flexible because they can be applied to different platforms without changing the hardware.

A software-based approach usually builds power/energy models to estimate the energy consumption at the CPU instruction level, program block level, process level, hardware component level or full system level. These models are built based on selection and optimization of power/energy indicators of a software or a hardware component of interests. Regarding the power/energy indicators type, software based approaches can be classified as system profile-based approaches or hardware performance counter (PMC) based approaches.

System profile or system events are a set of performance statistical information supplied by the operating system or a special software. These events can describe the current state of hardware, software, and operating system. For example, Joulemeter [33], tracks the energy consumption of a virtual machine using power/energy models for three system hardware components, i.e. CPU, memory and disk. The CPU energy model uses CPU utilization, the memory energy model uses the number of last level cache misses, while the disk energy model uses the bytes of data written to and read from the disk as inputs to their energy consumption estimates.

Hardware performance counters are a group of special registers that count the hardware-related activities within the computer systems. The PMC-based modelling [34] is implemented either with top-down or bottom-up approaches. The first do not depend on modelled architecture thus enabling a fast and an easy deployment [35], while the second depends on the underlying architecture and is therefore able to produce more informative, responsive and accurate power/energy models. The bottom-up approach enables a breakdown of the energy consumption per architectural components, but is more complex to deploy and less portable than the simpler top-down approach [36].

3. Use Cases and Experimental Setup. The use cases, presented in this section, have been used as test applications for the analyses of energy consumption using real-time power monitoring. The experimental computer comprised multi-core components, which are the main building blocks of the contemporary largescale computing systems, including those offered by the cloud infrastructure providers. The two use cases are of different types based on their scalability characteristics. The first is characterized by high scalability on the multi-core computer systems, and second is characterized by high communication costs, which limit the scalability. The use cases are also implemented with different parallelization techniques, master-slave parallelization and domain decomposition, and are consequently not equally sensitive to the computer architecture details, such as the amount of cache memory and the number of its levels.

Under the assumption that energy consumption scales linearly with the problem size on the selected use cases, we chose to experiment on small problems. With this assumption, the obtained results should also be representative for the larger problems. 
3.1. Maximum Clique Algorithm. For the first use case, we use an application that implements the parallel maximum clique algorithm [37], which is essentially a recursive program for traversing in a search tree under the branch and bound strategy. The algorithm takes an input graph and returns its maximal fully connected sub-graph. The maximum clique algorithm is characterized as a compute-bound algorithm and will thus benefit if more processing units are employed for its solution. It is parallelized by using threads for searching the tree branches asynchronously. The threads are synchronized only when accessing one global variable - the size of the current clique. On large graphs - these are graphs that take at least a few seconds to search through - the algorithm experiences a near-linear speedup on average.

As in all branch and bound approaches, the order in which the branches are searched influences the time required to find the optimal solution. In general, the concurrent search of several branches influences on which branches are searched and which not, which results in varying efficiency of the approach when used on various number of threads. The effects of parallel speedup, which influences the energy consumption greatly, can thus be hidden in the noise, generated by the varying branch and bound efficiency, which could make this algorithm tricky to evaluate.

For our experiments, a small graph named p_hat300-3 from the DIMACS collection of benchmark graphs for the maximum clique problem [38] was used. This graph comprises 300 vertices and 33390 edges, while its maximum clique spans over 36 of its vertices. The time required to find the maximum clique of p_hat300-3 on a single core of the experimental computer setup is about 1.3 seconds. A quality of this graph when searched by the selected maximum clique algorithm is also that it experiences relatively smooth speedup with almost constant branch and bound efficiency across the range of the experimented number of cores. By using this graph, we streamline the experiments that would otherwise have to be made on a large set of input graphs just to cover the average algorithm speedup.

3.2. WRF meteorological model. The second use case is a public domain Weather Research and Forecasting model (WRF-ARW) [39, 40]. This is a next-generation numerical weather prediction system designed to serve both operational forecasting and atmospheric research needs. The model, based on the input parameters and data, gives as an output a prediction of several atmospheric phenomena such as temperature, moisture, wind speed and direction, and precipitations. The model operates on a target geographical domain that is divided into a grid. The distance between the grid points is called resolution. To increase the accuracy of the forecast for a specific region, a smaller nested domain is defined within the main domain. The forecast is conducted at each grid point on multiple vertical levels (3D grid) for each time step.

In the experiments we compute a 6 -hour weather forecast for Croatia with one nested domain. The main domain consists of $100 \times 68$ points and the nested domain of $58 \times 61$ points with 18 and $6 \mathrm{~km}$ resolution, respectively, and 27 vertical levels. The time step is 2 minutes for the main, and 40 seconds for the nested domain, thus, the number of iterations/steps is 180 and 1080 for the main and nested domain, respectively. The total number of performed steps, for the 6 -hour simulation, is $1.36 \times 10^{8}$.

The model applies different model for parallelism: MPI, OpenMP and hybrid MPI+OpenMP, that enable execution on various computing architectures such as distributed and shared-memory (SMP) systems, clusters of SMPs, and vector and multiprocessors base systems. The parallelism in the model is achieved by decomposition of geographical domain (a domain on which the model simulation is run) into a $2 \mathrm{D}$ grid. The grid consists of patches, the rectangular sections of the model domain, each one allocated to a distributed memory node and executed by one MPI process. On a node level, each patch is further divided into tiles, which are the square sections of a patch, allocated to a shared-memory processor and each tile is executed by a single thread (i.e. OpenMP thread). Since our testing system is a shared-memory multi-core machine, only the OpenMP parallel version was considered. Thus, the domain is decomposed into 1 patch and the number of tiles equals to the number of threads (in our case up to 12 threads). The tiles are processed concurrently by different threads, but, if the tile size cannot fit into the CPU's cache memory, an additional communicational overhead is exhibited.

In contrast to the maximum clique algorithm, the performance of WRF is significantly leveraged by the communication and data exchange between the tiles that limit the scalability of the model and is thus concerned as a memory-bound algorithm. 
3.3. Experimental Setup. The performance of the use cases was evaluated in terms of execution time and the corresponding power consumption. All results are shown as the average of 15 experiments. The experimental computing system was running Ubuntu 12.04 server version OS and was composed of a dual CPU $2.3 \mathrm{GHz}$ Intel Xeon E5-2630, each of CPUs with six physical cores. Both applications were compiled with the GNU Compiler Collection (GCC), with parallelization flags -03 -march=native and with -lpthread for the MaxClique problem and -fopenmp for the WRF problem to exploit multi-threaded level of parallelism.

Use cases were executed on a varying number of cores up to the maximum available cores $n_{c}=[1 . .12]$, two CPU clock frequencies $f=[1.2 \mathrm{GHz}, 2.3 \mathrm{GHz}]$ which represent the minimum and maximum software settings for the clock frequency in the experimental computer, and two binding policies - bind and nobind. The bind policy stands for enforced binding of the $n$-th thread on the $n$-th core (as listed by the OS), and nobind denotes that binding is not enforced and thread management is completely up to the OS. It is worth noting that OS tends to migrate threads among cores often, which is expected to adversely effect performance by causing some unneeded cache misses in the core-local caches. On the other hand, the OS tends to distribute threads equally among all available CPUs, and thus make available to the application the CPU-local caches of all the installed CPUs. The effects of more caches can be difficult to predict, since they depend both on the application and on the values of parameters to the application, but more often than not, they correspond to a better performance [41].

Additional feature of core binding is that when all the required threads can be loaded onto cores of a singe CPU, the second CPU can be idle most of the time, unless loaded by the OS processes. Depending on the OS and the processor used, the idle processor can be put into one of its sleep states. The OS indexes cores of the first CPU as [1..6] and the cores of the second CPU as [7..12]. Therefore, while the enforced binding is used and the number of cores is set to 6 or lower, only the cores of the first CPU will be used by the application, enabling the OS to power down the second CPU. When binding is not enforced, OS tends to distribute the threads among cores of both CPUs, even when only 2 threads are requested. OS also switches threads among available cores at random intervals, which increases the number of cache misses, and consequently lowers the performance of the application. Binding of threads to processor cores is performed through the pthread API for the MaxClique case and through the likwid-pin tool, which comes with likwid Performance monitoring and benchmarking suite [42], for the WRF case.

Threads that are bound to cores are known to perform well by minimizing the low level cache flushing and reloading, which happens if threads are moved between cores. The effect is very application dependent though. In addition, when the program is executed on cores of multiple CPUs, instead of a single CPU, more cache is available to it, which can speedup the execution [41]. This effect is very application and problem dependent and rarely visible outside a narrow range of problem sizes. In our case there are only two CPUs available, which makes the possible effects of such experiment severely limited, therefore we do not test such arrangement at all.

\section{Experimental results.}

4.1. Maximum clique program. In this section we demonstrate the impacts of scaling the number of computational cores, CPU frequencies and binding policies on the performance and energy consumption on our use cases. The total execution time, power and energy consumption of the two use cases are shown in Figs. 4.1, 4.2 and 4.3, respectively. Since the measurement of the performance and energy consumption can be influenced by other running processes on the system (e.g. those managed by OS), we repeated each test 15 times (with the same experimental setup). The results presented in the figures are calculated as an average value of these repeated runs.

Figure 4.1(a) shows that the program for solving the maximum clique problem scales well on all test configurations, with the speedup between 8.1 and 9.4 on 12 cores for different clock frequencies and binding policies. The respective power consumption and energy consumption for the maximum clique problem are shown in Fig. 4.2(a), and Fig. 4.3(a), respectively. A closer look at Fig. 4.3(a) reveals that at higher frequencies, binding is significantly more energy efficient than leaving the thread management to OS, which spreads and moves the threads around the cores of both CPUs. Also, note that by binding the threads to physical cores, both the power and energy consumption are slightly reduced if the clock frequency is at its maximum while no significant difference is noticed if the clock frequency is at its minimum.

The evident finding, in general, is that running maximum clique program on less cores causes higher energy consumption. The second finding is that binding has positive effect on the power consumption but not on the 


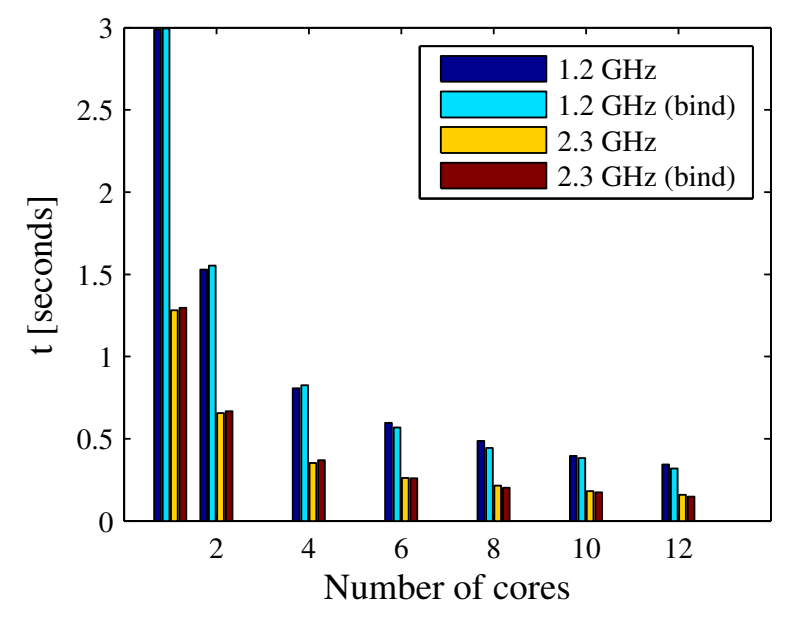

(a)

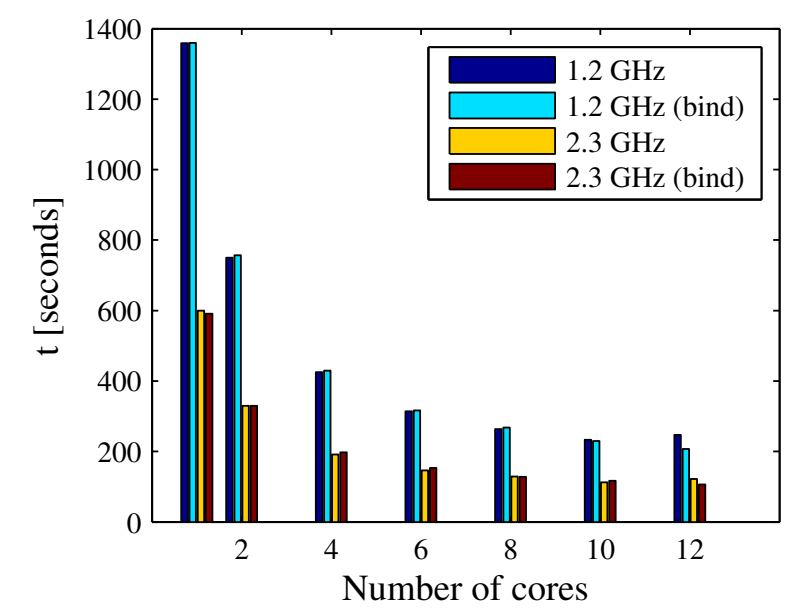

(b)

Fig. 4.1: Execution times for the maximum clique program (a) and the WRF model (b) as functions of the number of used cores, clock frequency, and binding policy.

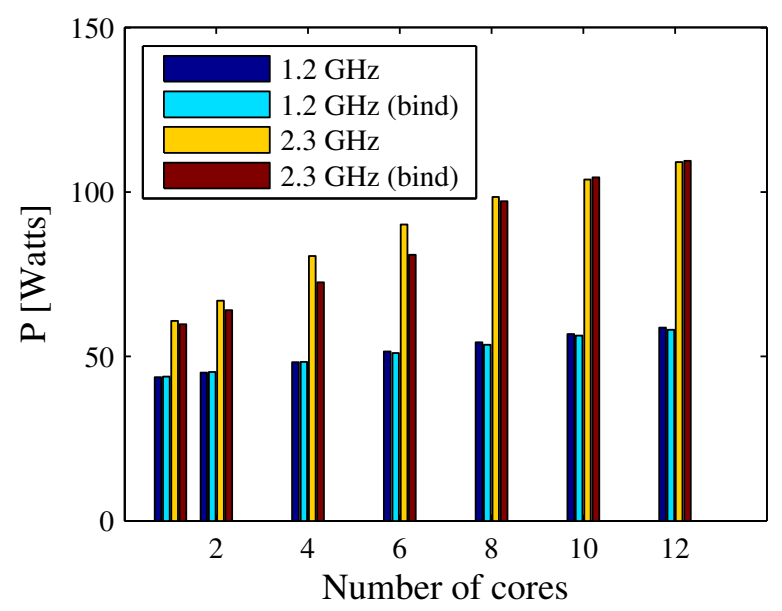

(a)

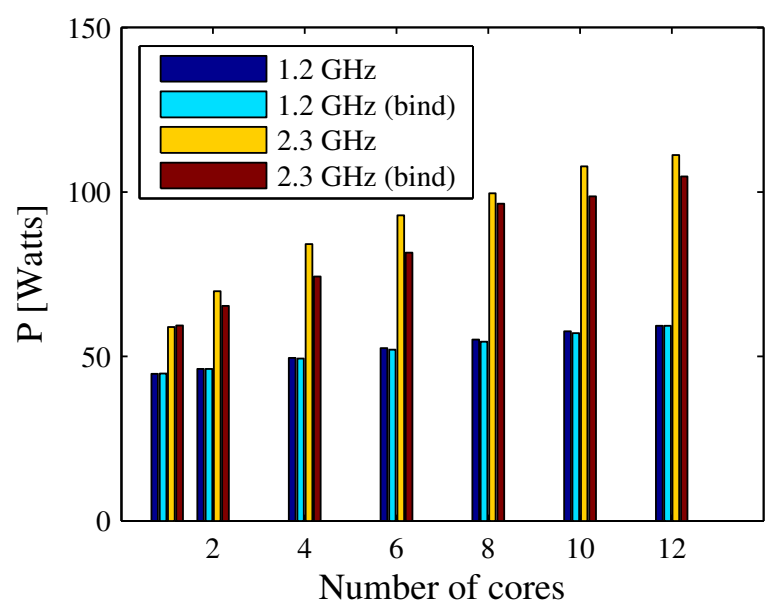

(b)

Fig. 4.2: Power consumption for the maximum clique program (a) and for the WRF model (b) as functions of the number of used cores, clock frequency, and binding policy.

execution time. The enforced binding only uses one CPU for up to 6 threads (cores indexed 1 through 6 are all on the first CPU), causing the second CPU to be idle and drawing less power, thus explaining the observed lower power consumption for a low number of cores. At more than 6 threads, both CPUs become active and the power consumption equalizes for both binding policies. The effects on execution time are both positive and negative. Binding threads to cores results in shorter execution times for the number of cores equal to or larger than 6 , which can be attributed to the better data locality. Binding also causes longer execution times for the number of cores between 1 and 4, even though the data locality should be better no matter the number of cores used. The bind policy restricting the cache access to only one CPU thus seem to have greater performance effect 


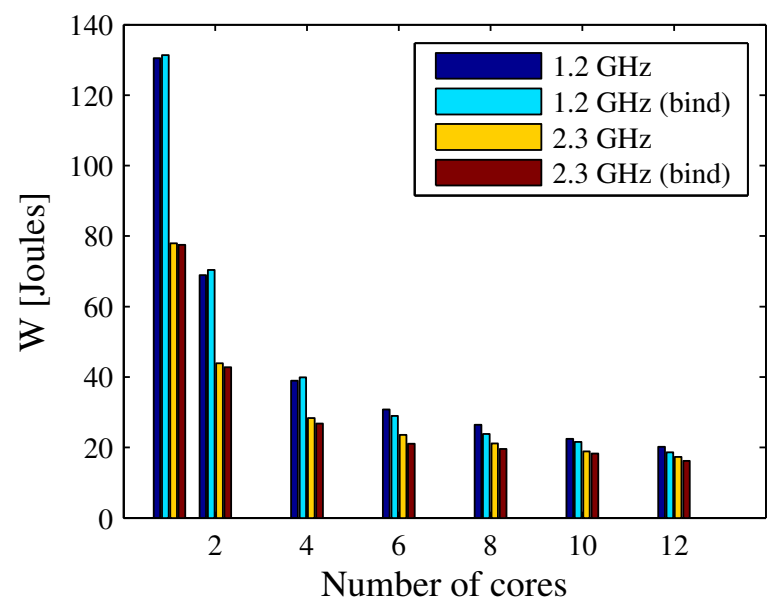

(a)

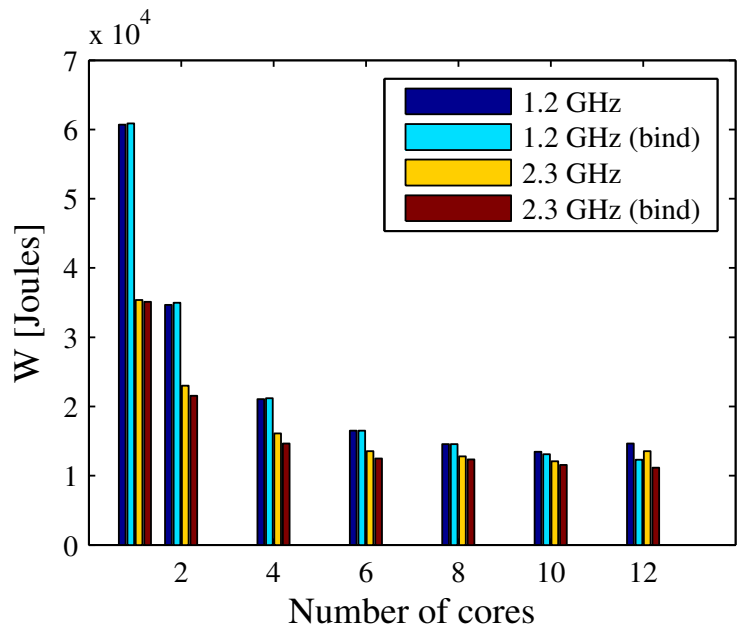

(b)

Fig. 4.3: Energy consumption for the maximum clique program (a) and for the WRF model (b) as a function of the number of used cores, clock frequency, and binding policy.

on $1-4$ cores than the improved data locality. Although the differences in performance are small, they are mostly statistically significant. Table 4.1 summarizes the differences in policy performance separately for each performance measure ( $\mathrm{t}, \mathrm{P}$, and $\mathrm{W}$ ), each tested frequency and each number of cores. For each set of parameter values, the performance is expressed by two statistics - ratio and p-value. Ratio is the mean ratio between the value obtained with bind policy and the value obtained with nobind policy. P-value is the p-value of the paired Student's $t$-test, on the null hypothesis that the performance is the same for both policies. Statistics are collected from 15 repeats of the maximum clique program execution for each set of input parameters (binding policy, number of cores, and clock frequency).

Table 4.1: Difference in performance of binding policies expressed as the statistics of bind policy relative to nobind policy for the maximum clique program.

\begin{tabular}{|c|c|c|c|c|c|c|c|c|}
\hline & cores & 1 & 2 & 4 & 6 & 8 & 10 & 12 \\
\hline \multicolumn{9}{|c|}{ Frequency $=1.2 \mathrm{GHz}$} \\
\hline \multirow[t]{2}{*}{$\mathrm{t}$} & ratio & 1.00 & 1.02 & 1.02 & 0.95 & 0.91 & 0.97 & 0.93 \\
\hline & p-value & ¡0.01 & ¡0.01 & ¡0.01 & ¡0.01 & ¡0.01 & ¡0.01 & ¡0.01 \\
\hline \multirow[t]{2}{*}{$\mathrm{P}$} & ratio & 1.00 & 1.00 & 1.00 & 0.99 & 0.99 & 0.99 & 0.99 \\
\hline & p-value & ¡0.01 & ¡0.01 & 0.38 & ¡0.01 & ¡0.01 & ¡0.01 & ¡0.01 \\
\hline \multirow[t]{3}{*}{$\mathrm{W}$} & ratio & 1.01 & 1.02 & 1.02 & 0.94 & 0.90 & 0.96 & 0.92 \\
\hline & p-value & ¡0.01 & ¡0.01 & $¡ 0.01$ & ¡0.01 & ¡0.01 & ¡0.01 & ¡0.01 \\
\hline & \multicolumn{8}{|c|}{ Frequency $=2.3 \mathrm{GHz}$} \\
\hline \multirow[t]{2}{*}{$\mathrm{t}$} & ratio & 1.01 & 1.02 & 1.05 & 0.99 & 0.94 & 0.96 & 0.93 \\
\hline & p-value & 0.01 & ¡0.01 & ¡0.01 & 0.14 & ¡0.01 & ¡0.01 & ¡0.01 \\
\hline \multirow[t]{2}{*}{$\mathrm{P}$} & ratio & 0.98 & 0.96 & 0.90 & 0.90 & 0.99 & 1.01 & 1.00 \\
\hline & p-value & 0.06 & ¡0.01 & ¡0.01 & ¡0.01 & ¡0.01 & 0.02 & 0.34 \\
\hline \multirow[t]{2}{*}{ W } & ratio & 0.99 & 0.97 & 0.94 & 0.89 & 0.93 & 0.97 & 0.94 \\
\hline & p-value & 0.63 & 0.05 & ¡0.01 & ¡0.01 & ¡0.01 & ¡0.01 & ¡0.01 \\
\hline
\end{tabular}


4.2. WRF program. Our second use case is the WRF model, a computationally-intensive problem whose performance is tackled by the communication speed and the size of the cache memory. As presented in Fig. 4.1(b), the scalability rate drops with the number of cores used, for both clock frequencies and binding policy configurations. The problem scales well up to 10 cores (speedup is between 4.3 and 5.9), but exhibits modest speedup on 12 cores only for variants using the bind policy, while the execution time for the nobind variants already increases. In contrast to the maximum clique algorithm, the speedup on 12 cores is a bit lower, and amounts between 4.9 and 6.5 due to a higher communication cost between the running threads. The explanation for that lays in the fact that when more cores are utilized, the tile size that can fit into the cache decreases, which increases the number of cache misses.

The corresponding power consumption and the energy consumption are illustrated in Figs. 4.2(b) and 4.3(b), respectively. Similar to the maximum clique problem, the tests show that the best energy-efficient strategy is to use all the available cores at their maximum performance. The same statistics as for the Maximum clique problem are gathered from 15 runs of the WRF model and summarized on Table 4.2. These show that for the clock frequency of $1.2 \mathrm{GHz}$, on one hand, binding has a very limited effect on execution time and power consumption (except on 12 cores), regardless of the binding policy. For the clock frequency of $2.3 \mathrm{GHz}$, on the other hand, binding the threads to physical cores reduces the power consumption considerably, while it does not change the execution times much.

Table 4.2: Difference in performance of binding policies expressed as the statistics of bind policy relative to nobind policy, for the WRF model

\begin{tabular}{|c|c|c|c|c|c|c|c|c|}
\hline & cores & 1 & 2 & 4 & 6 & 8 & 10 & 12 \\
\hline \multicolumn{9}{|c|}{ Frequency $=1.2 \mathrm{GHz}$} \\
\hline \multirow[t]{2}{*}{$\mathrm{t}$} & ratio & 1.00 & 1.01 & 1.01 & 1.01 & 1.02 & 0.98 & 0.84 \\
\hline & $\mathrm{p}$ value & ¡0.01 & ¡0.01 & ¡0.01 & ¡0.01 & ¡0.01 & 0.35 & ¡0.01 \\
\hline \multirow[t]{2}{*}{$\mathrm{P}$} & ratio & 1.00 & 1.00 & 1.00 & 0.99 & 0.99 & 0.99 & 1.00 \\
\hline & $\mathrm{p}$ value & 0.09 & 0.74 & 0.1 & ¡0.01 & ¡0.01 & ¡0.01 & 0.71 \\
\hline \multirow[t]{3}{*}{ W } & ratio & 1.00 & 1.01 & 1.01 & 1.00 & 1.00 & 0.98 & 0.84 \\
\hline & $\mathrm{p}$ value & 0.03 & ¡0.01 & ¡0.01 & 0.93 & 0.17 & 0.07 & ¡0.01 \\
\hline & \multicolumn{8}{|c|}{ Frequency $=2.3 \mathrm{GHz}$} \\
\hline \multirow[t]{2}{*}{$\mathrm{t}$} & ratio & 0.99 & 1.00 & 1.03 & 1.05 & 1.00 & 1.04 & 0.88 \\
\hline & $\mathrm{p}$ value & ¡0.01 & 0.94 & ¡0.01 & ¡0.01 & 0.85 & ¡0.01 & ¡0.01 \\
\hline \multirow[t]{2}{*}{$\mathrm{P}$} & ratio & 1.01 & 0.94 & 0.88 & 0.88 & 0.97 & 0.92 & 0.94 \\
\hline & $\mathrm{p}$ value & ¡0.01 & ¡0.01 & ¡0.01 & ¡0.01 & ¡0.01 & ¡0.01 & ¡0.01 \\
\hline \multirow[t]{2}{*}{ W } & ratio & 0.99 & 0.94 & 0.91 & 0.92 & 0.97 & 0.96 & 0.82 \\
\hline & $\mathrm{p}$ value & 0.13 & ¡0.01 & ¡0.01 & ¡0.01 & 0.02 & ¡0.01 & ¡0.01 \\
\hline
\end{tabular}

4.3. Energy consumption in detail. The energy consumption - calculated as power consumption times execution time and shown in Tables 4.1 and 4.2 - indicates that energy-wise, best strategy is using all available cores in combination with the thread binding strategy. This finding holds even though the WRF program exhibits relatively low speedup at 12 cores.

A significant observation on both use cases is the jump in the power consumption from 6 to 8 cores when threads are bound to cores. For up to 6 cores all threads are bound to one CPU, and the second CPU is in idle state, while for 8 or more cores the threads are distributed on both CPUs, which increases the power consumption in one large step (observe the jump between 6 and 8 cores bind versions, red color bars, in Fig. 4.2). Note that the energy consumption continues to decrease due to the lower computational time caused by adding additional cores. Once the second CPU is active, adding more cores again causes the execution time and the energy consumption to decrease. Therefore using only one processor with all available cores is worth considering for programs that might experience worse speedups than our use cases.

For the last result, we look at the statistics that we gather from the 15 repetitions of the same measurement. Figure 4.4 is the box plot of the WRF model energy consumption on the higher clock frequency $(2.3 \mathrm{GHz})$. It clearly shows the smaller variability and the lower number of outliers in the energy consumption if the bind 
policy is used, compared to the no-bind policy. Note that the results for the maximum clique use case on the higher clock frequency are very similar, while the results for both use cases on the low frequency display much less difference in binding policies. These results indicate the level of randomness inserted into the measurements by the OS regulated thread management.

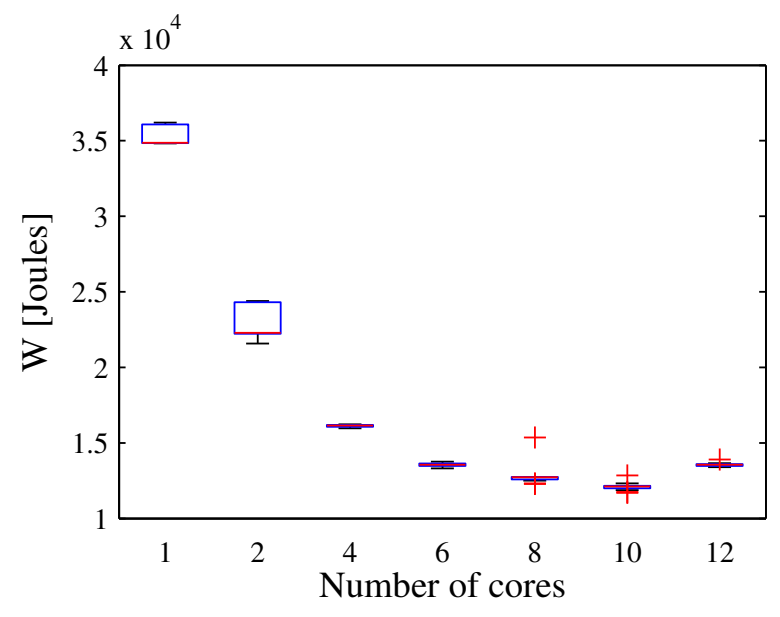

(a) No-bind

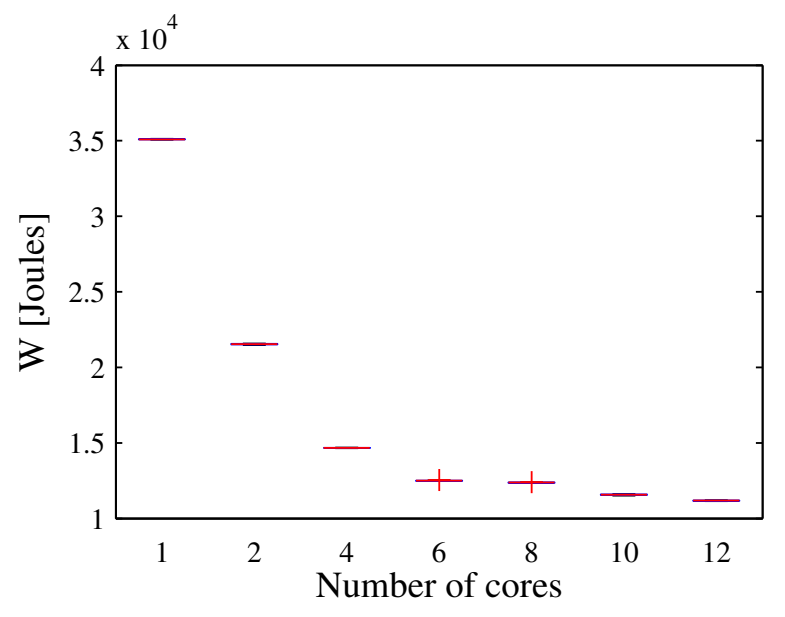

(b) Bind

Fig. 4.4: Energy consumption for WRF model as a function of the number of used cores and binding policy (a) the no-bind policy, and (b) the bind policy. Graphs show the median (red line), 25 and 75 percentiles (blue box), min and max values (blue whiskers), and outliers (red plus signs).

5. Discussion. A simple experimental study of a real-time power measurement in a multi-core computing system was presented. The energy consumption of two HPC parallel algorithms was measured, by the PCM library, as a function of the number of cores, the system clock frequency, and the binding policy. The main results of this research are listed below.

While we often believe that the two most influential factors on energy consumption are clock frequency and execution time, it turns out that execution time dominates clock frequency. The lower the execution time, the higher the energy efficiency, regardless of the clock frequency. This is caused by the relatively high baseline power consumption of the CPU, memory, and the rest of the system, which was not even considered in this study.

Second result shows that even less efficient parallel programs perform better, energy-wise, if executed on more cores. Note that the baseline consumption of the other computer parts, which was not included in the study, would again strengthen this finding even further. Of course, such a conclusion holds under the assumption that the computer is not multitasking - its unused cores actually remain idle and that the idle time of the computer can be ignored, which, for example, is not the case on computer resources rented for a fixed amount of time. A scenario where our findings hold is for computer resources that are rented on the cloud for the time of the execution only.

Third result is that by the same number of cores the energy consumption is lower if the cores are concentrated on a single CPU and not spread across more CPUs. It appears that the given CPU can enter a much more efficient power saving state if no cores are active than if even a single core is used. This, however, on our tests holds only for the high frequency and not for the low frequency, where the power consumption is not significantly different no matter how the threads are positioned on the CPUs. This could mean that the tested CPU could not throttle into a state with power consumption that is lower than when the clock frequency is the lowest. It should be noted though, that these results could be more profound - motherboard could use some additional energy for the inter-CPU communication when both CPUs are active, but motherboard is currently 
not monitored.

The fourth result is that the energy consumption for bind policy exhibits very small variation of the energy consumptions between runs as illustrated in Fig. 4.4. This enables a precise and accurate prediction of the energy consumption, especially if observed from the perspective of the cost reduction in utilizing public clouds with the pay-per-use policy. Small variation is possible because no flushing and reloading of cache are performed since threads are not moved among the cores. On the other hand, the nobind strategy, especially when applied on a smaller number of cores, exhibits a large difference between the best and the worst run. This can be explained by the fact that when the number of threads is small, OS is more likely to switch threads among cores and CPUs, causing cache flushing on various cache levels.

6. Conclusion. The obtained results show that idle power consumption of the CPU is significant. To achieve highest energy efficiency, the whole application should be executed in the shortest possible time. This can be achieved with cores at the highest possible system clock frequency, threads bound to cores and using the maximum number of cores at which the speedup is still satisfactory high. To apply the optimization of the energy consumption on general applications, a profiling of their speedup is required. In the tested cases, for example, each additional core causes about 4.5\% (mean of measured increases on the high clock frequency setting) higher power consumption of the CPU. Therefore, when deciding weather to use $n$ cores or $n+1$ cores, one should favour $n$ cores only if the relative decrease in execution time when raising the number of cores to $n+1$ is less than $4.5 \%$.

Aside from affecting the execution time, binding threads to cores also directly lowers the power consumption by limiting the number of cache flushes and main memory reads. This has been demonstrated in the WRF model case, but not in the maximum clique case, since the WRF model is a memory intensive application, while the maximum clique is more processing intensive. Caching is thus proved as another area, where more control should have to be given to the program designer, to be able to get maximal performance of the computer at the minimal energy cost.

To optimize the energy consumption more aggressively, one should be able to shut down the computing cores and banks of memory completely, or at least put them in a sleep state with a significantly lower power consumption. There are also some interesting possibilities of executing programs on multiple cores with heterogeneously set clock frequencies. In scenarios when computing resources are reserved for a predefined time frame - as in some Clouds services for example - measuring the power consumption during the whole time frame would be more to the point, instead of just for the time the program is executing. For such scenarios the role of the execution time in the energy consumption will be lowered and throttling the clock frequency might start to pay off. These are the topics of our current research and future work.

Acknowledgement. The work presented in this paper has been supported by the Ministry of Education, Science, Culture and Sport of the Republic of Slovenia and the Ministry of Science, Education and Sports of the Republic of Croatia under the bilateral project 'Energy Efficiency at Distributed Computing Systems', and partially supported by EU under the COST programme Action IC1305, 'Network for Sustainable Ultrascale Computing (NESUS)'.

\section{REFERENCES}

[1] G. L. Valentini, W. Lassonde, S. U. Khan, N. Min-Allah, S. A. Madani, J. Li, L. Zhang, L. Wang, N. Ghani, and J. KolOdZIEJ, An overview of energy efficiency techniques in cluster computing systems, Cluster Computing, 16 (2011), 1 , pp. $3-15$.

[2] J. Liu, D. Feld, Y. Xue, J. Garcke, T. Soddemann, Multicore Processors and Graphics Processing Unit Accelerators for Parallel Retrieval of Aerosol Optical Depth From Satellite Data: Implementation, Performance, and Energy Efficiency, IEEE Journal of Selected Topics in Applied Earth Observations and Remote Sensing, (2015), doi $=10.1109$ /JSTARS.2015.2438893, Article in Press.

[3] G. Kosec and P. Zinterhof, Local strong form meshless method on multiple Graphics Processing Units, CMES: Computer Modeling in Engineering and Sciences, 91 (2013), pp. 377-396.

[4] M. J. Flynn, O. Mencer, V. Milutinovic, G. Rakocevic, P. Stenstrom, R. Trobec, and M. Valero, Moving from Petaflops to Petadata, Commun. ACM, 56 (2013), pp. 39-42.

[5] A. C. Orgerie, L. Lefevre, and J. P. Gelas, Demystifying energy consumption in Grids and Clouds, Green Computing Conference, 2010 International, (2010), pp. 335-342. 
[6] A. Beloglazov, R. Buyya, Y. C. Lee, and A. Zomaya, A Taxonomy and Survey of Energy-Efficient Data Centers and Cloud Computing Systems, Advances in Computers, 82 (2011), 2, pp. 47-111.

[7] C. CaI, L. Wang, S. U. Khan, and J. TaO, Energy-aware high performance computing - A taxonomy study, in Proceedings of the International Conference on Parallel and Distributed Systems - ICPADS, 2011, pp. 953-958.

[8] J. Shuja, S. A. Madani, K. Bilal, K. Hayat, S. U. Khan, and S. Sarwar, Energy-efficient data centers, Computing, 94 (2012), pp. 973-994.

[9] J. Mukherjee And S. Raha, Power-aware Speed-up for Multithreaded Numerical Linear Algebraic Solvers on Chip Multicore Processors, Scalable Computing: Practice and Experience, 10 (2009), 2, pp. 217-228.

[10] A. Y. Zomaya and C. L. Young, Energy-efficient Distributed Computing, Awareness Magazine, January (2012).

[11] L. A. Barroso And U. Hölzle, The case for energy-proportional computing, Computer, 40 (2007), pp. 33-37.

[12] K. Bilal, S. U. K. Kashif, S. A. Madani, K. Hayat, M. I. Khan, N. Min-Allah, J. Kolodziej, L. Wang, S. Zeadally, and D. Chen, A survey on Green communications using Adaptive Link Rate, Cluster Computing, 16 (2012), 3, pp. 575-589.

[13] A. P. Bianzino, C. Chaudet, D. Rossi, And J.-L. Rougier, A survey of green networking research, IEEE Communications Surveys and Tutorials, 14 (2012), pp. 3-20.

[14] Y. LiU AND H. Zhu, A survey of the research on power management techniques for high-performance systems, Software Practice and Experience, 40 (2010), pp. 943-964.

[15] N. B. Rizvandi and A. Y. Zomaya, A Primarily Survey on Energy Efficiency in Cloud and Distributed Computing Systems. arXiv preprint, 2012.

[16] S. Libutti, G. Massari, P. Bellasi, W. Fornaciari, Exploiting performance counters for energy efficient co-scheduling of mixed workloads on multi-core platforms, ACM International Conference Prceeding Series, (2014), pp. 27-32.

[17] W. Lee, Y. Wang, M. Pedram,, Optimizing a Reconfigurable Power Distribution Network in a Multicore Platform, IEEE Transactions on Computer-Aided Design of Integrated Circuits and Systems, 34 (2015), 7, pp. 1110-1123.

[18] A. Mishra, A. K. Tripathi, Energy efficient voltage scheduling for multi-core processors with software controlled dynamic voltage scaling, Applied Mathematical Modelling, 38 (2014), 14, pp. 3456-3466.

[19] S. Pagani, J.-J. Chen, M. Li, Energy efficiency on multi-core architectures with multiple voltage islands, IEEE Transactions on Parallel and Distributed Systems, 26 (2015), 6, pp. 1608-1621.

[20] G. M. Tchamgoue, J. Seo, K. H. Kim, Y.-K. Jun, Compositional power-aware real-time scheduling with discrete frequency levels, Journal of Systems Architecture, 61 (2015), 7, pp. 269-281.

[21] C. Zhang, K. Huang, X. Cui, And Y. Chen, Power-aware programming with GPU accelerators, in Proceedings of the 2012 IEEE 26th International Parallel and Distributed Processing Symposium Workshops, IPDPSW 2012, 2012, pp. $2443-2449$.

[22] C. Lively, X. Wu, V. Taylor, S. Moore, H.-C. Chang, C. Yi Su, and K. Cameron, Power-aware predictive models of hybrid (MPI/OpenMP) scientific applications on multicore systems, Computer Science - Research and Development, 27 (2012), pp. 245-253.

[23] A. Holey, V. Mekkat, P.-C. Yew, A. Zhai, Performance-energy considerations for shared cache management in a heterogeneous multicore processor, ACM Transactions on Architecture and Code Optimization, 12 (2015), 1, pp. 1-29.

[24] V. Petrucci, O. Loques, D. Moss, R. Melhem, N. A. Gazala, S. Gobriel, Energy-efficient thread assignment optimization for heterogeneous multicore systems, ACM Transactions on Embedded Computing Systems, 14 (2015), 1, pp. 1-26.

[25] M. LI, Y. Zhao, Y. Si, Dynamic core allocation for energy-efficient thread-level speculation, Computational Science and Engineering (CSE), 2014 IEEE 17th International Conference on, 2014, pp. 682-689.

[26] R. Trobec, M. Depolli, K. Skala, and T. Lipic, Energy efficiency in large-scale distributed computing systems, in Information Communication Technology Electronics Microelectronics (MIPRO), 2013 36th International Convention on, May 2013, pp. 253-257.

[27] S. BenEDICT, Energy-aware performance analysis methodologies for HPC architectures - An exploratory study, Journal of Network and Computer Applications, 35 (2012), pp. 1709-1719.

[28] H. Chen And S. Weisong, Power Measuring and Profiling: the State of Art, andbook of Energy-Aware and Green Computing, pp. $649-674$.

[29] Watts up. https://www.wattsupmeters.com, 2014.

[30] M. A. Viredaz and D. A. Wallach, Power evaluation of Itsy version 2.3, tech. report, 2000.

[31] D. C. Snowdon, S. M. Petters, And G. Heiser, Power measurement as the basis for power management, Proceedings of the 2005 Workshop on Operating System Platforms for Embedded Real-Time Applications, Palma, Mallorca, Spain, 2005.

[32] A. Y. Zomaya And Y. C. Lee, Energy Efficient Distributed Computing Systems, Wiley-IEEE Computer Society Pr, Hoboken, NJ, USA, 1st ed., 2012.

[33] A. Kansal, F. Zhao, J. Liu, N. Kothari, and A. A. Bhattacharya, Virtual machine power metering and provisioning, in Proceedings of the 1st ACM Symposium on Cloud Computing, SoCC '10, 2010, pp. 39-50.

[34] R. Bertran, M. Gonzalez, X. Martorell, N. Navarro, and E. Ayguade, Counter-based power modeling methods: Topdown vs bottom-up, The Computer Journal, 56 (2013), pp. 198-213.

[35] W. L. Bircher And L. K. John, Complete system power estimation using processor performance events, IEEE Transactions on Computers, 61 (2012), pp. 563-577.

[36] R. BERTRAn, A systematic methodology to generate decomposable and responsive power models for CMPs, IEEE Transactions on Computers, 62 (2013), 7, pp. 1289-1302.

[37] J. Konc AND D. JANEŽIČ, An improved branch and bound algorithm for the maximum clique problem, Match, 58 (2007), pp. $569-590$.

[38] D. J. Johnson And M. A. TrICK, eds., Cliques, Coloring, and Satisfiability: Second DIMACS Implementation Challenge, Workshop, October 11-13, 1993, American Mathematical Society, Boston, MA, USA, 1996. 
[39] J. Michalakes, S. Chen, J. Dudhia, L. Hart, J. Klemp, J. Middlecoff, and W. Skamarock, Development of a Next Generation Regional Weather Research and Forecast Model, in Developments in Teracomputing: Proceedings of the Ninth ECMWF Workshop on the Use of High Performance Computing in Meteorology, 2001, pp. 269-276.

[40] J. Michalakes, J. Dudhia, D. Gill, T. Henderson, J. Klemp, W. Skamarock, and W. Wang, The Weather Research and Forecast Model: Software Architecture and Performance, in Proceedings of the Eleventh ECMWF Workshop on the Use of High Performance Computing in Meteorology, World Scientific, 2005, pp. 156-168.

[41] G. Kosec, M. Depolli, A. Rashkovska, and R. Trobec, Super Linear Speedup in a Local Parallel Meshless Solution of Thermo-fluid Problems, Comput. Struct., 133 (2014), pp. 30-38.

[42] Likwid. https://github.com/rrze-likwid/likwid, 2015.

Edited by: Dana Petcu

Received: July 15, 2015

Accepted: September 30, 2015 